\title{
Evaluation of Recombinant SRS3 Antigen for Diagnosis of Toxoplasmosis by Enzyme-Linked Immunosorbent Assay
}

\author{
Seyedeh Mahya Marashiyan, ${ }^{1}$ Fatemeh Moradian, ${ }^{1}$ Geita Saadatnia,,${ }^{2,}$ and Majid Golkar ${ }^{3}$ \\ ${ }^{1}$ Department of Basic Sciences, Sari Agricultural Sciences and Natural Resources University, Sari, Iran \\ ${ }^{2}$ Department of Biotechnology, Iranian Research Organization for Science and Technology (IROST), Tehran, Iran \\ ${ }^{3}$ Molecular Parasitology Laboratory, Department of Parasitology, Pasteur Institute of Iran, Tehran, Iran \\ "Corresponding author: Geita Saadatnia, Department of Biotechnology, Iranian Research Organization for Science and Technology (IROST), Tehran, Iran. Tel/Fax: \\ +98-2156276636, E-mail: gitasaadat@gmail.com
}

Received 2015 December 19; Revised 2016 October 31; Accepted 2016 November 06.

\begin{abstract}
Background: Infections by the protozoan parasite Toxoplasma gondii (T. gondii) are widely prevalent in human and animals. SRS3 is a member of SRS antigen family (glycosylphosphatidylinositol-linked proteins), which is structurally related to the highly immunogenic surface antigen SAG1. The SAG family of proteins can cover the surface of T. gondii bradyzoites and tachyzoites.

Objectives: The aim of this study was to evaluate the recombinant SRS3 protein for diagnosis of toxoplasmosis by enzyme-linked immunosorbent assay.

Methods: Indirect immunoglobulin G (IgG) enzyme-linked immunosorbent assays (ELISAs) with recombinant SRS3 and SAG1 proteins of $T$. gondii were developed to evaluate the diagnosis of infection in pregnant women.

Results: The results showed that sensitivity and specificity were respectively $84.12 \%$ and $92 \%$ for recombinant protein SRS3 and $92 \%$ and $96 \%$ for rSAG1.

Conclusions: Recombinant proteins SRS3 and rSAG1 are promising antigens in diagnostic assays for detection of specific antibodies against T. gondii.
\end{abstract}

Keywords: Toxoplasma gondii, Recombinant Protein SRS3, Recombinant Protein SAG1, ELISA

\section{Background}

Toxoplasma gondii (T. gondii) is an obligate intracellular parasite capable of infecting virtually any warm-blooded animal including human and Livestock. Toxoplasma infections are widespread and can lead to severe disease in individuals with an immature or suppressed immune system. Accurate diagnosis of acute toxoplasmosis during pregnancy permits timely treatment and prevents or attenuates congenital toxoplasmosis. The immune system in normal individuals is able to control the infection and hence, most healthy infected persons have no clinical disease due to infection. However, even in healthy individuals, the immune system is not able to eradicate the infection, and allows T. gondii to persist as latent bradyzoites in the host (1).

A crucial parameter for parasite attachment is surface coat. Toxoplasma utilizes an evolutionally expressed collection of structurally related proteins, which yet antigenically are defined as surface proteins, to adhere and enter the host cell. This adherence additionally absorbs the immune reaction against the tachyzoite stage that seemingly controls the pathogenicity of infection (2).

In toxoplasmosis, IgG antibody produced by the immune system remains in the bloodstream for a long time.
The external antigens of $T$. gondii that act in the processes of host cell attachment and host immune elusion are controlled by a SRS (SAG1-related sequence) family of proteins. SRS proteins are expressed in a stage-specific manner. SAG1, SAG2A, SAG2B, SAG3, SRS1, SRS2, and SRS3 are mostly expressed on the tachyzoite surface. The tachyzoite surface comprises five major antigens that the most common of them is SAG1 (surface antigen 1). Acute infection, relating to the fast dividing form or 'tachyzoite', is normally organized by the immune system, with a main role for interferon-g (IFN-g)-dependent mechanisms. But, the parasite can differentiate into a 'bradyzoite' state, which can insist in the host within cysts that are seemingly resistant to the strong immune response induced by tachyzoites (3, $4)$.

All SRS (SAG1-related sequence) proteins seem to be glycosylphosphatidylinositol (GPI)-anchored (5). It has been shown that tachyzoite surface is controlled by SAG1, SAG2A, SAG3, SRS1, SRS2, SRS3, and several less greatly expressed SRSs (5-8). SRS3 proteins which have shown in-vivo expression as well as great sensitivity and specificity with individual serum samples deserve further study on their probable role in pathogenesis of the toxoplasmosis (9). Identification of T. gondii infection depends on serological tests such

Copyright (c) 2016, Infectious Diseases and Tropical Medicine Research Center. This is an open-access article distributed under the terms of the Creative Commons Attribution-NonCommercial 4.0 International License (http://creativecommons.org/licenses/by-nc/4.0/) which permits copy and redistribute the material just in noncommercial usages, provided the original work is properly cited. 
as enzyme-linked immunosorbent assay (ELISA) that predominantly utilize the total antigens of the parasite. Therefore, they require large amount of the parasite in the peritoneal fluid of mice or cell cultures that makes them expensive and laborious methods; there is also the possibility of contamination of non-parasitic antigens derived from mice or cell cultures $(10,11)$.

Recent developments have been made in recombinant antigens of T. gondii which are less expensive and have constant quality in different batches. The efficacy of recombinant antigens for improving performance of serologic testing of $T$. gondii infection, differentiating between acute and chronic infection, and protecting mice cells against the infection has been investigated in numerous studies (12-14). SRS3 tachyzoite antigen as a surface antigen may be beneficial in the recognition of infection and vaccination. A few studies have been conducted for these purposes.

\section{Objectives}

This study aimed to investigate the antigenicity of SRS3 recombinant protein for diagnosis of Toxoplasma infection by enzyme-linked immunosorbent assay.

\section{Methods}

\subsection{Sample Collection and Infection Diagnostic Test}

Serum samples were collected from 30 patients with acute toxoplasmosis and 33 women whose serodiagnostic test results indicated the infection acquired in the past. All acute and chronic sera were tested for Toxoplasma-specific IgG, IgM, and IgG avidity using commercial ELISA kits (Euroimmun, Lubeck, Germany). Sera with IgM+ and low IgG avidity were considered to be from patients with probable acute infection; while those with IgM+ and high IgG avidity were considered to be from chronically infected individuals. Also, 25 controls which tested negative for both $T$. gondii IgG and IgM were used in this study. Samples were collected in the Pasteur Institute of Iran.

\subsection{Expression and Purification of rSRS3}

Recombinant SRS3 antigen was produced in our previous study, briefly as follows: SRS3 gene was cloned in expression vector pET-28b (+) and transformed into E.coli rosetta (DE). Transformed bacteria were cultured in 500 $\mathrm{mL}$ of broth media for 24 hours. Then, recombinant protein was extracted from bacteria and prepared for purification using affinity chromatography Ni-NTA. After that, purified rSRS3 was analyzed by $12 \%$ sodium dodecyl sulfatepolyacrylamide gel electrophoresis (SDS-PAGE) and western blotting.

\subsection{Determination of rSRS3 and rSAG1 Antigenicity by ELISA}

In the process of rSRS3 antigenicity determination, we applied recombinant SAG1 that was already developed in our laboratory and has been checked for ELISA detection (15). In this study, the same serum samples were used for rSRS3 and rSAG1. Recombinant proteins SAG1 and SRS3 were prepared at concentration of $0.5 \mu \mathrm{L} / \mathrm{mL}$ in coating buffer $\left(\mathrm{Na}_{2} \mathrm{CO}_{3}, \mathrm{NaHCO}_{3}, \mathrm{NaCL} \mathrm{pH}=9.6\right)$ and added to each well of ELISA plates that coated with $100 \mu \mathrm{L}$ of the buffer. The plates were incubated overnight at $4^{\circ} \mathrm{C}$. The wells were washed two times with washing buffer (PBS1X, Tween $0.1 \%, \mathrm{pH}=7.2$ ) and incubated with blocking solution (PBS1X, Tween $0.1 \%$. BSA $1 \%$ ) at $37^{\circ} \mathrm{C}$ for 1 hour; then, the wells were washed three times using washing buffer. After that, $100 \mu \mathrm{L}$ of human sera with dilution of $1: 100$ was added and incubated for 1 hour at room temperature. Next, it was washed again with washing buffer, and 100 $\mu \mathrm{L}$ of horseradish peroxidase-conjugated goat antihuman IgG (Sigma) at the dilution of 1/30000 was added to each well for 40 minute at room temperature. After 3 times washing, the enzymatic activity was made using the substrate tetramethylbenzidine. Finally, 10 minutes of incubation at room temperature was elapsed and the reaction was stopped by adding $100 \mu \mathrm{L}$ of phosphoric acid, and the optical density (OD) was measured at $450 \mathrm{~nm}$ using a ELISA reader (Flow, Les Ullis, France) with a reference wavelength of $630 \mathrm{~nm}$.

\subsection{Statistical Analyses}

Statistical analyses were performed using the SPSS statistical software version 16 . The cut-off was calculated with the mean absorbance readings of negative sera plus 2 SD (standard deviation). Pearson test was used to analyze the correlation between SRS3-IgG-ELISA and SAG1-IgGELISA, and among SRS3-IgG-ELISA, Euroimmun IgG ELISA, and SAG1-IgG-ELISA. Antibody levels were compared among chronic and acute sera using t-test and Mann-Witheney.

\section{Results}

Antigen activity of rSRS3 was detected in Western-blot analysis (data submitted for publication). A single band was observed in membrane for acute and chronic phases of toxoplasma infection. While pulled acute and chronic sera reacted with rSRS3, negative sera failed to recognize any protein. Obtaining a stronger band in acute sample indicated that the antigenicity of rSRS3 is more in acute phase (data submitted for publication). In this study, all of the 88 samples from 3 different groups of sera were tested using rSRS3-IgG-ELISA and rSAG1-IgG-ELISA. The best conditions were chosen to create the greatest difference between the positive and negative sera in optical density. 
Optical density of SRS3 and SAG1 recombinant proteins was measured by ELISA reader at $450 \mathrm{~nm}$. In order to determine the sensitivity and specificity, the cut-off value was calculated with the mean plus 2 standard deviations of optical density (OD) values of 25 serum samples with no Toxoplasma infection (cut-off value for rSRS3 $=0.314$ and for rSAG1 = 0.456). A serum sample was considered positive if its optical density was higher than the determined cut-off value.

Using the cut-off value for SRS3, 2 out of 25 negative sera had absorption above the cut-off (specificity of 92\%), and 10 out of 63 positive sera had absorption below the cutoff (sensitivity of $84.12 \%$ ). According to the optical density, sensitivity to acute and chronic sera was obtained as $83.33 \%$ and $81.81 \%$, respectively. The scatter plot of optical density of acute, chronic, and negative sera in SRS3-IgG- ELISA is shown in Figure 1.

Figure 1. Scatter Plot of Optical Density of Acute, Chronic, and Negative Sera in SRS3IgG-ELISA

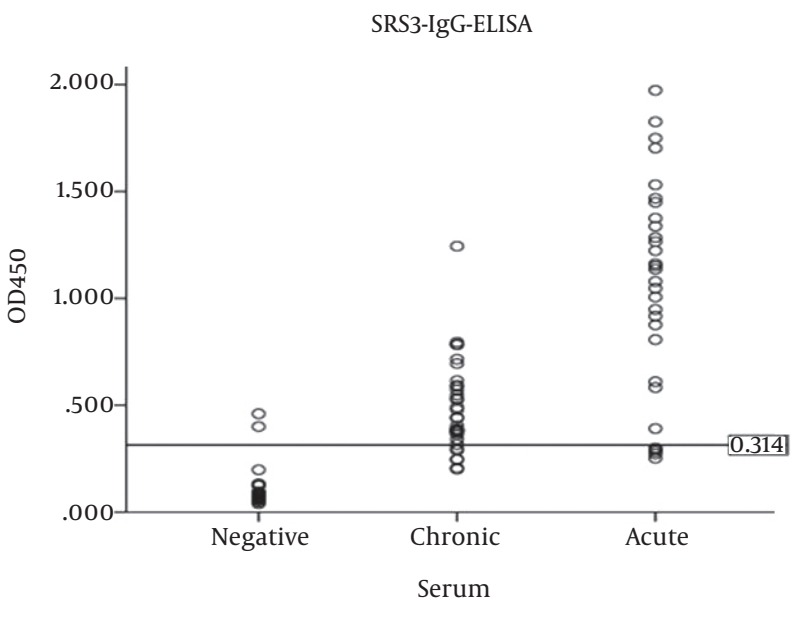

The black line shows a cut-off for diagnosis of toxoplasma infection.

Using the cut-off value for SAG1, 1 out of 25 negative sera had optical density above the cut-off; as a result, specificity was $96 \%$. The sensitivity was obtained as $92 \%$, because 58 out of 63 positive sera had absorption above the cut-off. According to optical density, sensitivity to acute and chronic sera was obtained as $96.6 \%$ and $87.87 \%$, respectively. The scatter plot of optical density of acute, chronic, and negative sera in SAG1-IgG- ELISA is illustrated in Figure 2.

The mean absorption of acute and chronic sera for rSRS3 was significant $(\mathrm{P}=0.000$, Mann-Whitney test) as the same as rSAG1 $(\mathrm{P}=0.011$, t-test $)$. The comparison of SRS3-IgGELISA for chronic sera with SAG1-IgG-ELISA revealed a strong correlation among them $(\mathrm{r}=0.708, \mathrm{P}=0.000$, Pearson test $)$. In contrast, SRS3-IgG-ELISA for acute sera showed a weak,
Figure 2. Scatter Plot of Optical Density of Acute, Chronic, and Negative Sera in SAG1IgG-ELISA

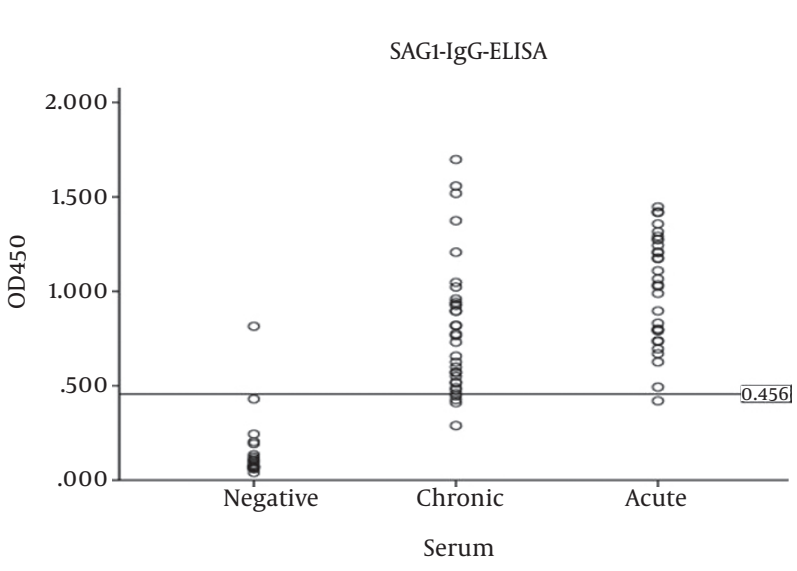

The black line shows a cut-off for diagnosis of toxoplasma infection.

non-significant correlation with SAG1-IgG- ELISA ( $\mathrm{r}=0.349$, $\mathrm{P}=0.0 .058$, Pearson test).

The comparison of SRS3-IgG-ELISA for 27 chronic sera with Euroimmun IgG ELISA showed a weak, non-significant correlation among them $(\mathrm{r}=0.362, \mathrm{P}=0.64$, Pearson test $)$. In contrast, SRS3-IgG-ELISA for acute sera showed a significant correlation with Euroimmun IgG ELISA ( $\mathrm{P}=0.041, \mathrm{r}$ $=0.375)$. The comparison of SAG1-IgG-ELISA for 27 chronic sera with Euroimmun IgG ELISA showed a strong, significant correlation among them $(\mathrm{r}=0.634, \mathrm{P}=0.000$, Pearson test) and also showed a strong and significant correlation between them $(r=0.622, P=0.000)$.

\section{Discussion}

The gold standard for detection of T. gondii is Dye-test; however, usual diagnosis of the infection relies on serological tests, such as enzyme-linked immunosorbent assay (ELISA), which mainly uses the whole antigens of the parasite. Recent advances have been made in generating recombinant antigens of $T$. gondii. The major advantage of using recombinant antigens for diagnosis of the infection is the ability to combine antigens in a single detection kit. Also, the use of recombinant antigens leads to better standardization of testing and reduce the cost of production. These considerations are very important when only one serum sample is available from a suspected patient (15). Furthermore, usefulness of recombinant antigens for improving performance of serologic tests for diagnosis of $T$. gondii infection, particularly for distinguishing between acute and chronic phases, has been approved (12-14).

Sine finding an accurate and sensitive diagnostic method for opportunistic infections such as toxoplasmo- 
sis is useful and necessary, in recent years various molecular diagnostic tools have been developed for the detection of T. gondii. Fallahi et al. in 2014 for the first time applied loop-mediated isothermal amplification (LAMP) and nested-PCR using the RE and B1 gene to detect T. gondii in blood samples; however, the LAMP method has not been widely introduced for detection of toxoplasmosis, yet (16, 17). SRS3 tachyzoite antigen is a surface antigen that can be useful in the diagnosis of infection and vaccination (18). The selection of SRS3 genes in this study was based on our previous research, whereby in-vivo induced antigen technology (IVIAT) was applied to identify antigens which are expressed in-vivo during T. gondii infection. In that study, sera from individuals with toxoplasmosis were used and promising results were obtained for SRS3 (9). However, so far no report is available regarding the assessment of the antigenicity of this protein.

IgG and IgM antibodies are major isotopes that are used to diagnose toxoplasmosis. Determination of antitoxoplasma IgG was the most common method used to trace antibodies in individuals. Immunodominant surface antigen of T. gondii, SAG1, is debated a consequential antigen for progress of effective diagnostic tests and propose vaccines $(19,20)$. SAG1 was selected in this study since in numerous studies it is reported as a good candidate for toxoplasma detection $(13,18,21-24)$. Our results about the sensitivity and specificity of IgG-ELISA- rSAG1 was consistent with those reported by Khanaliha et al. (22) and Jalallou et al. (23). In a previous study by Selseleh et al. (24), an ELISA was described using recombinant SAG1 for detecting IgM and IgG antibodies. The sensitivity and specificity were $87 \%$ and 95\% for IgM and 93\% and 95\% for IgG, respectively. Our results are consistent with their findings.

SRS3 protein showed high sensitivity and specificity when tested with individual serum samples (9). We found that the sensitivity and specificity of rSRS3 in IgG-ELISA were $84.12 \%$ and $92 \%$, respectively. This is partly close to the sensitivity obtained with recombinant protein SAG1 for acute and chronic sera. The mean optical density of acute sera ( $\mathrm{P}=0.011$ for SAG1, t-test, and $\mathrm{P}=0$ for SRS3, MannWhitney test) was significantly higher than the mean of chronic sera.

The mean optical density of acute and chronic sera showed significant differences; however, according to the mean optical density in acute and chronic sera of recombinant protein SRS3, mean absorbance was two times in acute and chronic sera, and the results indicated that the protein expression was more in the acute than chronic phase.

\subsection{Conclusion}

The present study is the first report of the efficacy of recombinant protein SRS3 for the diagnosis of toxoplasmosis using ELISA. The recombinant proteins showed high efficacy for the detection and differentiation of acute and chronic Toxoplasma infection. Previous diagnostic markers are recognized by false positive and negative results while the recombinant proteins did not show this drawback in this study. Thus, recombinant SRS3 is useful in the diagnosis of toxoplasmosis and can be a marker of acute infection.

\section{Acknowledgments}

This original research was performed in the Iranian research organization for science and technology and Pasteur institute of Iran. The authors are thankful for providing facilities and assistance.

\section{Footnotes}

Authors' Contribution: Geita Saadatnia and Majid Golkar contributed to the study conception, design, analysis, and interpretation of the data. Fatemeh Moradian contributed to drafting the manuscript and critical revision and finalizing the manuscript. Seyedeh Mahya Marashiyan collected and analyzed the data. Geita Saadatnia also contributed to revising the paper.

Funding/Support: Partial funding of this project was provided by Ministry of Health and Medical Education, Iran and EMGEN fund provided under grant through Deputy of Research and Technology, Ministry of Health and Medical Education, Iran, grant No: 20134.

\section{References}

1. Yap GS, Sher A. Cell-mediated immunity to Toxoplasma gondii: initiation, regulation and effector function. Immunobiology. 1999;201(2):240-7. doi: 10.1016/S0171-2985(99)80064-3. [PubMed: 10631573].

2. Boothroyd JC, Hehl A, Knoll LJ, Manger ID. The surface of Toxoplasma: more and less. Int J Parasitol. 1998;28(1):3-9. doi: 10.1016/S00207519(97)00182-3. [PubMed: 9504330].

3. Darcy F, Santoro F. Parasitic infections and the immune system. San Diego, California: Academic Press Inc; 1994. pp.163-20.

4. Sims T, Hay J, Talbot I. An electron microscope and immunohistochemical study of the intracellular location of Toxoplasma tissue cysts within the brains of mice with congenital toxoplasmosis. British J Experiment Pathol. 1989;90(3):317-25.

5. Lekutis C, Ferguson DJ, Grigg ME, Camps M, Boothroyd JC. Surface antigens of Toxoplasma gondii: variations on a theme. Int J Parasitol. 2001;31(12):1285-92. [PubMed: 11566296]. 
6. Dzierszinski F, Mortuaire M, Cesbron-Delauw MF, Tomavo S. Targeted disruption of the glycosylphosphatidylinositol-anchored surface antigen SAG3 gene in Toxoplasma gondii decreases host cell adhesion and drastically reduces virulence in mice. Mol Microbiol. 2000;37(3):574-82. doi: 10.1046/j.1365-2958.2000.02014.x. [PubMed: 10931351].

7. Jung C, Lee CY, Grigg ME. The SRS superfamily of Toxoplasma surface proteins. Int J Parasitol. 2004;34(3):285-96. doi: 10.1016/j.ijpara.2003.12.004. [PubMed: 15003490].

8. Rollins SM, Peppercorn A, Young JS, Drysdale M, Baresch A, Bikowski MV, et al. Application of in vivo induced antigen technology (IVIAT) to Bacillus anthracis. PLoS One. 2008;3(3):1824. doi: 10.1371/journal.pone.0001824. [PubMed: 18350160].

9. Amerizadeh A, Idris ZM, Khoo BY, Kotresha D, Yunus MH, Karim IZ, et al. Identification of Toxoplasma gondii in-vivo induced antigens by cDNA library immunoscreening with chronic toxoplasmosis sera. Microb Pathog. 2013;54:60-6. doi: 10.1016/j.micpath.2012.09.006. [PubMed: 23044055].

10. Gross U, Holpert M, Goebel S. Impact of stage differentiation on diagnosis of toxoplasmosis. Ann Ist Super Sanita. 2004;40(1):65-70. [PubMed: 15269454].

11. Gatkowska J, Hiszczynska-Sawicka E, Kur J, Holec L, Dlugonska H. Toxoplasma gondii: an evaluation of diagnostic value of recombinant antigens in a murine model. Exp Parasitol. 2006;114(3):220-7. doi: 10.1016/j.exppara.2006.03.011. [PubMed: 16707125].

12. Piergili Fioretti D. [Problems and limitations of conventional and innovative methods for the diagnosis of Toxoplasmosis in humans and animals]. Parassitologia. 2004;46(1-2):177-81. [PubMed: 15305712].

13. Pietkiewicz H, Hiszczynska-Sawicka E, Kur J, Petersen E, Nielsen HV, Paul M, et al. Usefulness of Toxoplasma gondii recombinant antigens (GRA1, GRA7 and SAG1) in an immunoglobulin G avidity test for the serodiagnosis of toxoplasmosis. Parasitol Res. 2007;100(2):333-7. doi: 10.1007/s00436-006-0265-1. [PubMed:16896649].

14. Suzuki LA, Rocha RJ, Rossi CL. Evaluation of serological markers for the immunodiagnosis of acute acquired toxoplasmosis. J Med Microbiol. 2001;50(1):62-70. doi: 10.1099/0022-1317-50-1-62. [PubMed: 11192507].

15. Pietkiewicz H, Hiszczyriska-Sawicka E, Kur J, Petersen E, Nielsen HV, Stankiewicz M, et al. Usefulness of Toxoplasma gonidi specific recombinant antigens in serodiagnosis of human toxoplamosis. Clin Diag- nostic laboratory Immunol. 2004;42(4):1779-81.

16. Fallahi S, Seyyed Tabaei SJ, Pournia Y, Zebardast N, Kazemi B. Comparison of loop-mediated isothermal amplification (LAMP) and nestedPCR assay targeting the RE and B1 gene for detection of Toxoplasma gondii in blood samples of children with leukaemia. Diagn Microbiol Infect Dis. 2014;79(3):347-54. doi: 10.1016/j.diagmicrobio.2014.02.014. [PubMed: 24792838].

17. Fallahi S, Arab Mazar Z, Ghasemian M, Haghighi A. Challenging loopmediated isothermal amplification (LAMP) technique for molecular detection of Toxoplasma gondii. Asian Pacific J Tropical Med. 2015;3:366-72. doi: 10.1016/S1995-7645(14)60345-X.

18. Manger ID, Hehl AB, Boothroyd JC. The surface of Toxoplasma tachyzoites is dominated by a family of glycosylphosphatidylinositolanchored antigens related to SAG1. Infect Immun. 1998;66(5):2237-44. [PubMed: 9573113].

19. Buffolano W, Beghetto E, Del Pezzo M, Spadoni A, Di Cristina M, Petersen $\mathrm{E}$, et al. Use of recombinant antigens for early postnatal diagnosis of congenital toxoplasmosis. J Clin Microbiol. 2005;43(12):5916-24. doi: 10.1128/JCM.43.12.5916-5924.2005. [PubMed: 16333076].

20. Handman E, Remington JS. Antibody responses to toxoplasma antigens in mice infected with strains of different virulence. Infect Immun. 1980;29(1):215-20. [PubMed: 7399703].

21. Allahyari M, Mohabati R, Babaie J, Amiri S, Siavashani ZJ, Zare M, et al. Production of in-vitro refolded and highly antigenic SAG1 for development of a sensitive and specific Toxoplasma IgG ELISA. J Immunol Methods. 2015;416:157-66. doi: 10.1016/j.jim.2014.11.012. [PubMed: 25437069].

22. Khanaliha K, Motazedian MH, Kazemi B, Shahriari B, Bandehpour M, Sharifniya Z. Evaluation of recombinant SAG1, SAG2, and SAG3 antigens for serodiagnosis of toxoplasmosis. Korean J Parasitol. 2014;52(2):137-42. doi: 10.3347/kjp.2014.52.2.137. [PubMed: 24850956].

23. Jalallou N, Bandepour M, Khazan H, Haghighi A, Abdollahi S, Kazemi B. Recombinant SAG1 Antigen to Detect Toxoplasma gondii Specific Immunoglobulin G in Human Sera by ELISA Test. Iran J Parasitol. 2010;5(2):1-9. [PubMed: 22347238].

24. Selseleh MM, Keshavarz H, Mohebali M, Shojaee S, Modarressi M, Eshragian M, et al. Production and Evaluation of Toxoplasma gondii Recombinant Surface Antigen 1 (SAG1) for Serodiagnosis of Acute and Chronic Toxoplasma Infection in Human Sera. Iran J Parasitol. 2012;7(3):1-9. [PubMed: 23109955]. 